\title{
Scaling the Topology of Symmetric, Second-Order Planar Tensor Fields
}

\author{
Xavier Tricoche, Gerik Scheuermann, and Hans Hagen \\ University of Kaiserslautern, P.O. Box 3049, 67653 Kaiserslautern, Germany \\ E-mail: $\{$ tricoche |scheuer|hagen $\}$ @informatik.uni-kl.de
}

\begin{abstract}
Tensor fields can be found in most areas of physics and engineering sciences. Topology-based methods provide efficient means for visualizing symmetric, second-order, planar tensor fields. Yet, for tensor fields with complicated structure typically encountered in turbulent flows, topology-based techniques lead to cluttered pictures that confuse the interpretation and are of little help for analysts. In this paper, we present a hierarchical method that merges close degenerate points into one. This results in a simplified topology and a clarified depiction, though globally maintaining the qualitative properties of the original data. The whole process can be seen as a scaling of the topological information that neglects details of small scale and only retains their structural aspect in the large. We extend here previous work dealing with planar vector fields. Results are demonstrated on a computational fluid dynamics(CFD) dataset.
\end{abstract}

\section{Introduction}

Tensors are the language of mechanics. Therefore, tensor field visualization is a challenging issue for scientific visualization. Scientists and engineers need techniques that enable both qualitative and quantitative analysis of tensor data sets resulting from experiments or numerical simulations. A topology-based visualization method of symmetric, second-order tensor fields in two dimensions has been designed for that purpose [1]. It focuses on one of the two eigenvector fields corresponding to the minor or major eigenvalues. Like the vector case, the displayed graph consists of so-called degenerate points (where both eigenvalues are equal) connected by particular integral curves, the separatrices. This technique proved to be suitable for tensor fields with simple structure because the extracted topology contains few degenerate points and separatrices, leading to a comprehensible structure description. However, for tensor fields with complicated structure (like those encountered in turbulent flows, for instance), topology-based methods result in cluttered pictures that confuse the interpretation.

We present in this paper a method that merges close degenerate points into one, which simplifies the topology and clarifies its depiction, though globally maintaining the qualitative properties of the original data. It is the extension to the tensor case of previous work on vector fields [2]. We assume a planar, piecewise bilinear interpolated, symmetric, second-order tensor field over a curvilinear grid. The degenerate points are determined first. A clustering strategy is then applied to the resulting set of degenerate points. This leads to a grid partition into cell clusters such that 
the distance between degenerate points in each cluster is below a user-prescribed threshold. After this, we merge the degenerate points lying in each cluster to get the desired scaling effect. Finally, we determine and integrate the resulting separatrices.

The structure of the paper is as follows. Fundamental notions of tensor field topology are briefly presented in section 2 . The formula governing eigenvalue and eigenvector computation are proposed in section 3 . The clustering strategy is the same as for vector fields and has been presented earlier [2]. We bring back its basic principle in section 4 . Once clusters have been defined, we achieve a local grid deformation which merges singular points, as explained in section 5. The a posteriori analysis of the resulting local topological structure is detailed in sections 6,7 . At last, results are shown in section 8 on a swirling jet simulation with evolving turbulence: The topology is clarified, the separatrices easier to track while the significant structural features have been preserved.

\section{Topology of Tensor Fields}

Based upon the concept of vector field topology, the topology of symmetric secondorder 2D tensor fields has been introduced recently [1]. Basically, it is defined as the topology of one of the two (bidirectional) eigenvector fields. Since tensor field topology has been treated in very few publications so far, we give next the necessary definitions.

A real two-dimensional symmetric matrix $M$ has always two (not necessarily distinct) real eigenvalues $\lambda_{1} \leq \lambda_{2}$ with associated (orthogonal) eigenvectors $\boldsymbol{e}_{\mathbf{1}}$ and $e_{2}$ :

$$
\forall i \in\{1,2\}, M \boldsymbol{e}_{\boldsymbol{i}}=\lambda_{i} \boldsymbol{e}_{\boldsymbol{i}}, \text { with } \boldsymbol{e}_{\boldsymbol{i}} \in \mathbb{R}^{2} \text { and } \boldsymbol{e}_{\boldsymbol{i}} \neq \mathbf{0}
$$

(Note that an eigenvector as neither norm nor orientation for every multiplication of an eigenvector with a non-zero scalar results in another eigenvector.) Therefore, we write a symmetric, second order planar tensor field, i.e. a matrix-valued function that associates every position of a subdomain of $\mathbb{R}^{2}$ with a symmetric matrix:

$$
T:(x, y) \in U \subset \mathbb{R}^{2} \mapsto T(x, y)=\left(\begin{array}{c}
\alpha(x, y) \beta(x, y) \\
\beta(x, y) \gamma(x, y)
\end{array}\right) .
$$

One can define a major (resp. minor) eigenvector field, at each position of the domain, as the eigenvector related to the major (resp. minor) eigenvalue of the tensor field. One defines major (resp. minor) tensor lines as the curves everywhere tangent to the major (resp. minor) eigenvector field. Consequently, these curves have no orientation. Yet, this definition only holds outside locations where both eigenvalues are equal and thus the eigenvectors cannot be uniquely determined. These positions are called degenerate points (or singular points). Thus, at a degenerate point, the tensor value is always a diagonal matrix of the form $\alpha I_{2}$, which is used in practice to compute the position of the singularities. In the linear case, they appear in two possible types: Trisector or wedge point (see Fig. 1). The classification of a degenerate point can be done by the computation of its index (see [4], p. 105): The 


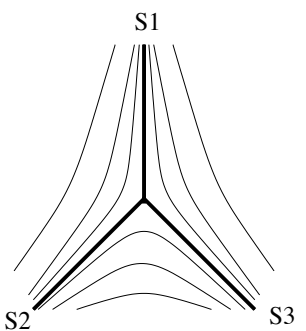

TRISECTOR

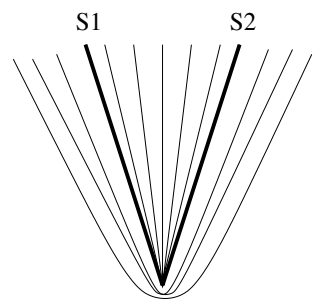

WEDGE POINTS

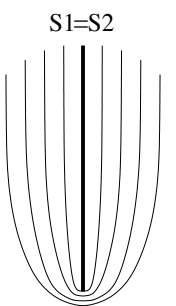

T S

Fig. 1. First Order Degenerate Points

index of a degenerate point of a symmetric tensor field is the number of counterclockwise rotations made by the eigenvectors along a closed, non self-intersecting curve around the degenerate point that encloses no other singularity, when traveling once in a counterclockwise direction along this curve. A trisector point has index $-\frac{1}{2}$ while a wedge point has index $+\frac{1}{2}$.

In the general (non-linear) case, a degenerate point can have two generic types: center type (no tensor line reaches the singularity, so every tensor line in its vicinity is closed and rotates around the singularity location) or non-center type (at least, one tensor line reaches the singularity, forming one or more curvilinear sectors): See Fig. 2. In the non-center case, the characterization is based upon position and type

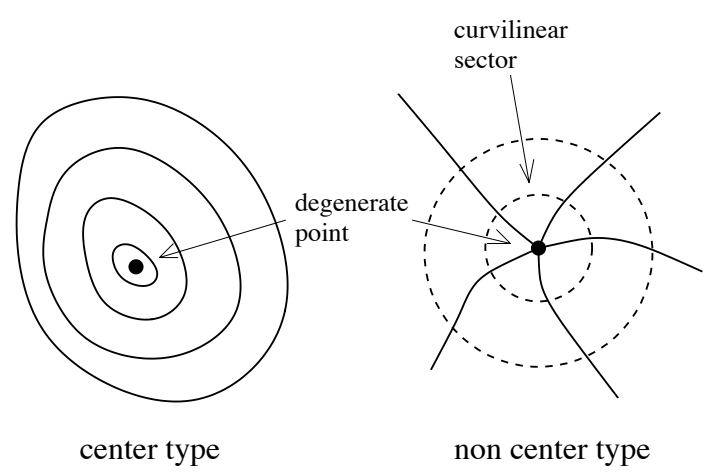

Fig. 2. Center and Non-Center Type for a Degenerate Point

of the curvilinear sectors. These sectors have three possible natures: Hyperbolic, parabolic or elliptic (see Fig. 3). Separatrices are defined as streamlines bounding hyperbolic sectors. Now, with the definitions above, the topology of an eigenvector field is defined as the graph formed by all degenerate points and by the separatrices connecting them. (Closed orbits, known from vector field topology, are very rare in practice). 


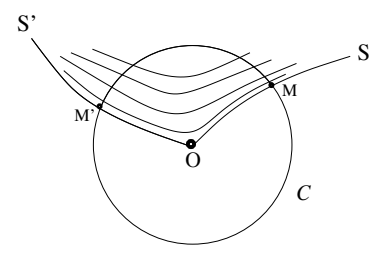

(a) Hyperbolic

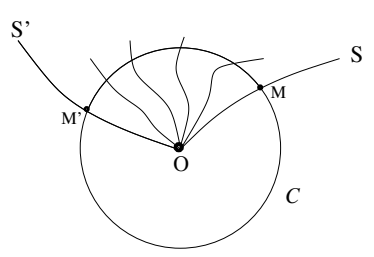

(b) Parabolic

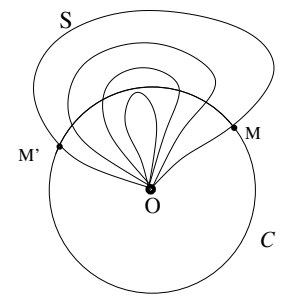

(c) Elliptic

Fig. 3. Sector Natures

\section{Eigenvectors and Eigenvalues Computation}

As said previously, the depiction of the topology of a symmetric, second-order tensor field is based upon the computation of its eigenvalues and eigenvectors. As a preliminary step, we first compute the so-called deviator [3] of the considered tensor field, defined as follows. Let $T$ be a symmetric, second-order planar tensor field

$$
T:=\left(\begin{array}{cc}
\alpha & \beta \\
\beta & \gamma
\end{array}\right),
$$

where $\alpha, \beta$ and $\gamma$ are scalar functions in the coordinates $x$ and $y$ of the Euclidean space $\mathbb{R}^{2}$. Denoting $\operatorname{tr}(T)=\alpha+\beta$ the trace of $T$, the isotropic part of $T$ is given by

$$
J:=\frac{1}{2} \operatorname{tr}(T) I_{2}
$$

and the deviator of $T$ is then defined by

$$
D:=T-J=\left(\begin{array}{rr}
\delta & \beta \\
\beta & -\delta
\end{array}\right), \text { where } \delta=\frac{\alpha-\gamma}{2}
$$

Now, the topology of a symmetric, second-order tensor field is the same as the topology of its deviator field because both fields have the same eigenvector fields, as we show next. By definition of an eigenvalue $\lambda$ and its corresponding eigenvector $\boldsymbol{e} \neq \mathbf{0}$, one has

$$
T \boldsymbol{e}=\lambda \boldsymbol{e} .
$$

Using the notations above, this is equivalent to

$$
\left(D+\frac{1}{2} \operatorname{tr}(T) I_{2}\right) \boldsymbol{e}=\lambda \boldsymbol{e},
$$


that is

$$
D e=\left(\lambda-\frac{1}{2} \operatorname{tr}(T)\right) \boldsymbol{e} .
$$

Thus, setting $\mu=\lambda-\frac{1}{2} \operatorname{tr}(T), \mu$ is eigenvalue of the deviator $D$ and $\boldsymbol{e}$ is the corresponding eigenvector. Furthermore, if one denotes by $\mu_{i}$ the eigenvalue related to $\lambda_{i}$, one gets $\mu_{1}<\mu_{2}$ if $\lambda_{1}<\lambda_{2}$. This proves the equivalence of both topologies.

For this reason, we will only consider matrices of deviator type in the following, which simplifies the calculus.

Now, the eigensystem (1) is singular (for there exist non-zero solutions) which is equivalent to

$$
\operatorname{det}\left(T-\lambda I_{2}\right)=0 .
$$

For each position on the plane, this is a quadratric polynomial equation that we use in practice to compute the eigenvalues. With the deviator, one gets

$$
\lambda_{1,2}= \pm \sqrt{\delta^{2}+\beta^{2}}
$$

Except in the special case where the deviator is diagonal (i.e. $\beta=0$ ), solving the singular eigensystem (1) leads to the following expressions (with the same notations). The eigenvectors are $\boldsymbol{e}_{1,2}:=\left(u, v_{1,2}\right)^{T}$ with

$$
u=\beta \text { and } v_{1,2}=-\delta \pm \sqrt{\delta^{2}+\beta^{2}}
$$

If the deviator is diagonal, the eigensystem becomes trivial: The eigenvalues are $\delta$ and $-\delta$ and the corresponding eigenvectors are the basis vectors of the Euclidean coordinate system: $(1,0)^{T}$ and $(0,1)^{T}$.

\section{Clustering}

We recall now the scheme we used in previous work[2] for grouping grid cells together in order to get a domain decomposition into cell clusters that contain only close singularities. The clustering process is monitored by the proximity of the singularities contained in a cluster. For simplicity, we deal in this section with a curvilinear grid mapped in computational space to a rectilinear grid. We denote by $P_{1}, \ldots, P_{m}$ the positions of the $m$ singularities lying inside a particular cluster. We want to minimize the approximation error of these $m$ singularities by a single point, where this point (or cluster center) $Q$ is chosen to be the best approximation (for a particular norm) of the singularities (see Fig. 4).

The corresponding error is

$$
S=\frac{\sum_{j=1}^{m} \omega_{j}\left\|P_{j}-Q\right\|}{\sum_{j=1}^{m} \omega_{j}}
$$




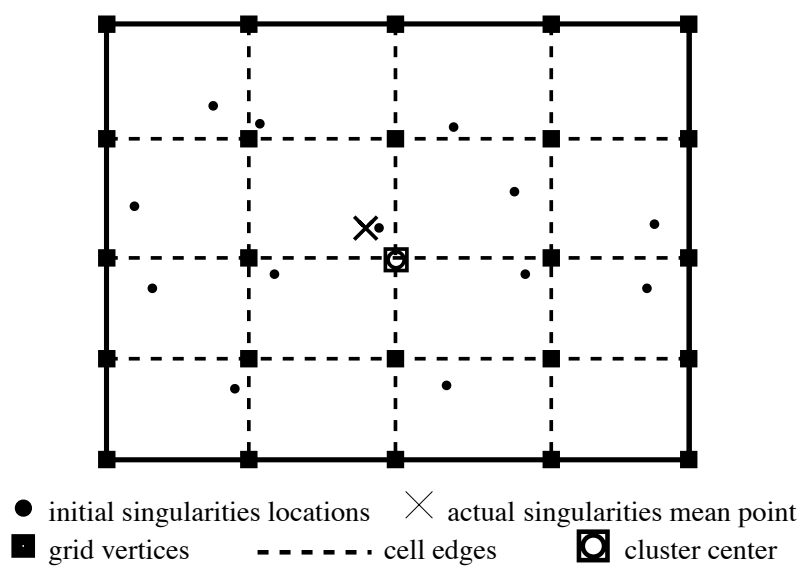

Fig. 4. Cluster singularities and cluster center

where $\omega_{i}$ is the weight associated with the $i$ th singularity: We have set these weights to 1 in the following for simplicity but these values can be used to introduce more flexibility in the method, in particular by privileging degenerate points with interesting numerical or qualitative properties, based upon user-defined criteria. The aim of our clustering scheme is now to get a domain decomposition into clusters that all fulfill an approximation criterion, that is that all have an error value smaller than a specified threshold (which is the only parameter of our method).

If a cluster does not satisfy the given error criterion, we split it into two subclusters. To do this, we need to introduce the projected variances associated with a given cluster:

$$
V_{i}=\sum_{j=1}^{m} \omega_{j}\left(P_{j}^{i}-Q^{i}\right)
$$

where $i \in 0,1$ is the considered coordinate axis $\left(P_{j}=\left(P_{j}^{0}, P_{j}^{1}\right)\right)$.

Now, considering the whole grid as initial cluster, the method is as follows.

Step 1. Take as cluster center the best vertex approximation of all cluster singular points.

Step 2. Compute the approximation error $\mathrm{S}$. If ( $\mathrm{S}>$ THRESHOLD) go to step 3 . Otherwise stop.

Step 3. Compute the coordinate axis with largest projected variance (i.e. $\max (\mathrm{V} 0, \mathrm{~V} 1))$. 
Step 4. Create 2 subclusters by splitting the

cluster at an edge polyline through $Q$

perpendicular to the selected

coordinate axis.

For each cluster, go to step 1 .

Step 4 justifies the need for a cluster center to be a grid vertex. As a matter of fact, when splitting a cluster, we keep processing cell groups in the form of Fig. 5. To

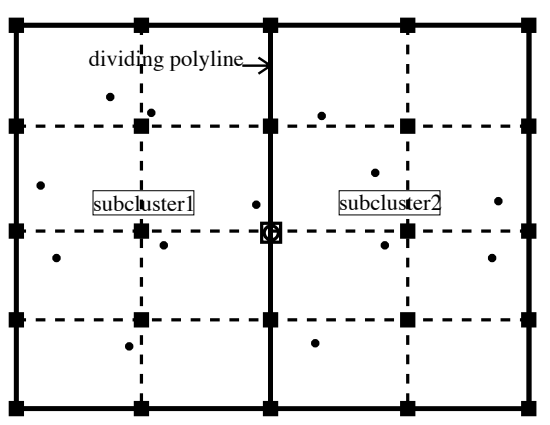

Fig. 5. Cluster splitting

ensure that the algorithm terminates, we require the existence of a best singularity mean point approximation by a grid vertex that does not lie on the cluster boundary, as additional criterion.

\section{Local Topology Deformation}

In each cluster, we aim at locally simulating the fusion of all preexisting singularities while preserving consistency with the original global topology. Practically, we must remove the degenerate points, replace them by a single one and let the cluster boundary unchanged. Like the vector case, we achieve it as follows: We first remove all quadrilateral cells in the cluster. Then we add a new vertex at the cluster center, associated with a degenerate tensor value. At last, we cover the resulting empty domain by linear interpolated triangles joining the new vertex to those on the cluster boundary. Yet, contrary to the vector case, a degenerate tensor value is not unique and may be any isotropic tensor (c.f. section 3). Now, as we chose to deal with a deviator tensor field, the only possible choice in our case is a zero matrix that we associate with the new vertex.

\section{6 “Parallel" Positions Localization}

Once such an artificial degenerate point has been created, we need to determine its local structure to find the positions of its separatrices. As a matter of fact, separatri-

\footnotetext{
${ }^{1}$ in computational space
} 
ces are integral curves that bound the so-called hyperbolic sectors of a singularity (see section 2). For that reason, boundary curves and sector types must be found. As the singularity lies inside a piecewise linear domain, one can show that its structure may be fully identified on the piecewise linear edges on the boundary of its containing cluster. For each such edge, one has the following configuration: One is given a segment $\overline{\left|M_{1} M_{2}\right|}$ with linear parameterization $t \in[0,1]$ on which a linear varying, symmetric, second-order (deviator) tensor field is defined. Furthermore, one is given the cluster center position $\Omega$ that does not lie on $\overline{\left|M_{1} M_{2}\right|}$. Now, we seek on $\overline{\left|M_{1} M_{2}\right|}$ the positions $M(t)$ where the vector $\Omega M$ is parallel to the eigenvectors, that is

$$
\Omega M \wedge e=0
$$

with a linear parameterization of the considered edge that can be written

$$
M(t)=(x(t), y(t))^{T}=(1-t) M_{1}+t M_{2}, \quad t \in[0,1] .
$$

Supposing $\beta(t) \neq 0$, the "parallel" condition becomes then (with the notations of section 3)

$$
\left|\begin{array}{lr}
x(t) & \beta(t) \\
y(t) & -\delta(t) \pm \sqrt{\delta(t)^{2}+\beta(t)^{2}}
\end{array}\right|=0
$$

( $\beta$ and $\delta$ being linear functions in $t$ ). Finally, the expression above is equivalent to the following system.

$$
\left\{\begin{array}{l}
\beta(t)=0 \\
\text { or } \\
x(t)^{2} \beta-y(t)^{2} \beta(t)-2 x(t) y(t) \delta(t)=0
\end{array}\right.
$$

As $x(t), y(t), \beta(t)$ and $\delta(t)$ are all linear functions in $t$, the second equation is a cubic polynomial that we solve with a standard analytic method to obtain the required "parallel" positions.

\section{Sector Type Identification}

Because of the sign indeterminacy, the sector type identification cannot be obtained by the method described in the vector case. As a matter of fact, the distinction between hyperbolic and elliptic type is impossible without additional information. For that reason, we use the tensor index (see section 2): Between two consecutive "parallel" positions, we compute the angle variation of the considered eigenvector field (see Fig. 6). Depending on the sector type, one gets

- $\Delta \alpha=\theta \quad$ in the parabolic case

- $\Delta \alpha=\theta-\pi$ in the hyperbolic case

- $\Delta \alpha=\theta+\pi$ in the elliptic case

which enables a sector type identification. When a hyperbolic sector is found, its boundary curves are then integrated to form the topological graph. 


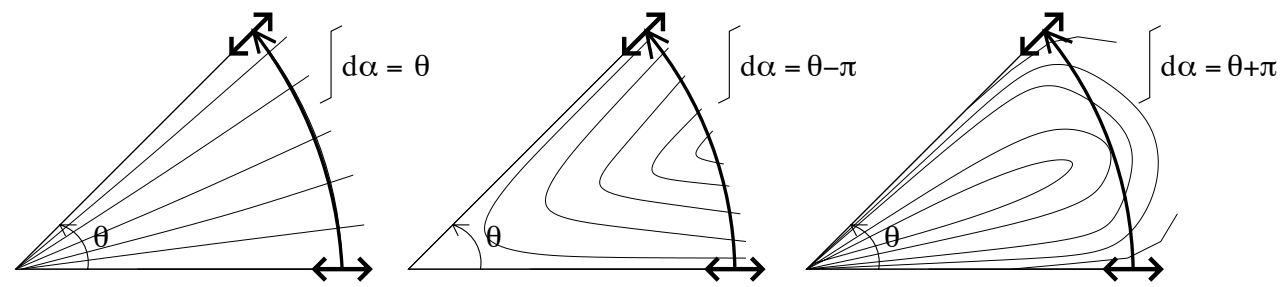

Fig. 6. Angle variation in the parabolic, hyperbolic and elliptic case

\section{Results}

We show the results of our method applied on the rate of strain tensor field of a swirling jet simulation. The dataset is almost axisymmetric, a property that is well preserved by our algorithm. The grid is structured and has $124 \mathrm{x} 101$ cells ranging from 0 to 9.87 in $\mathrm{x}$, resp. -3.85 to 3.85 in $\mathrm{y}$. The original topology presents 61 degenerate points and 131 separatrices. It is shown in Fig. 7.

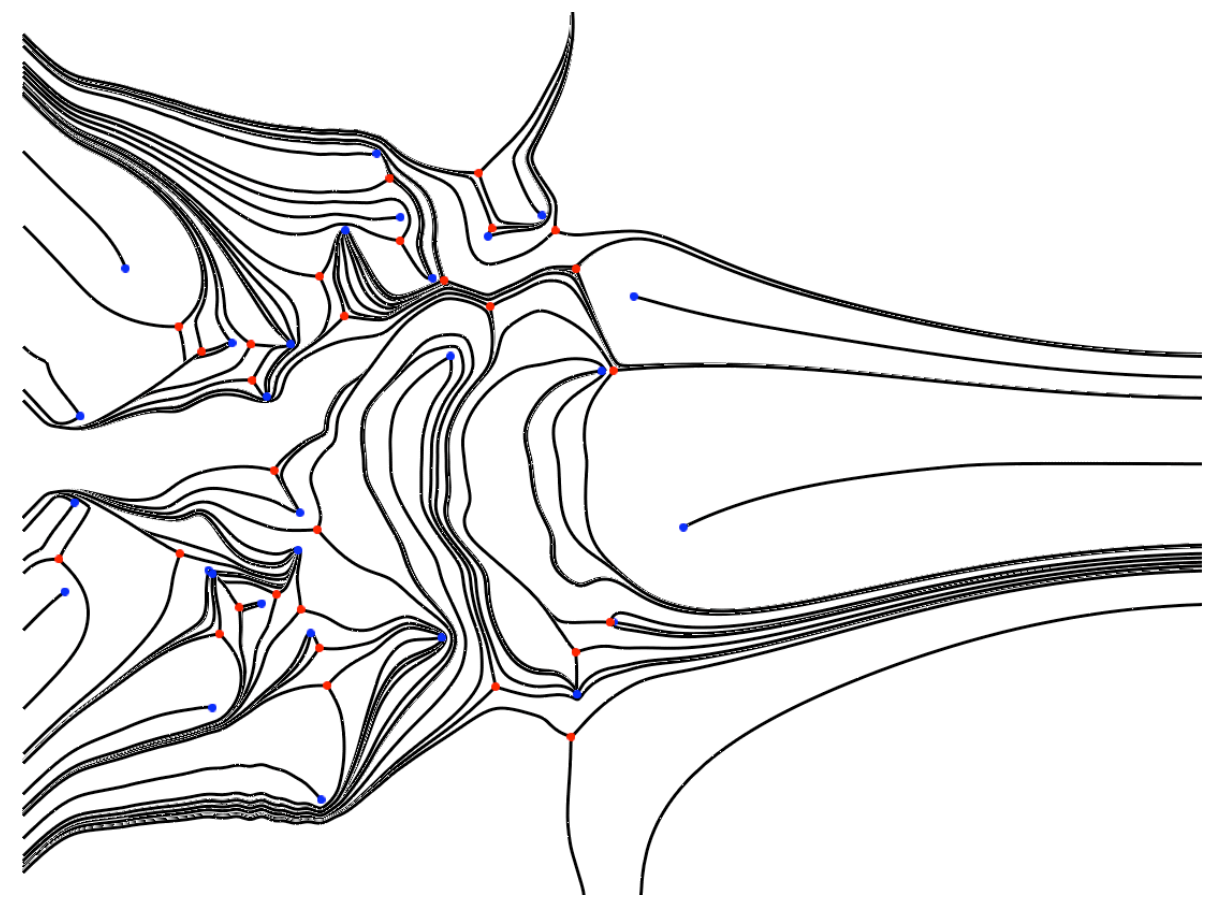

Fig. 7. Original topology

We start scaling with threshold $=0.2$. The resulting topology contains 44 singularities (13 of which being non-linear) and 101 associated separatrices: See Fig. 8. 


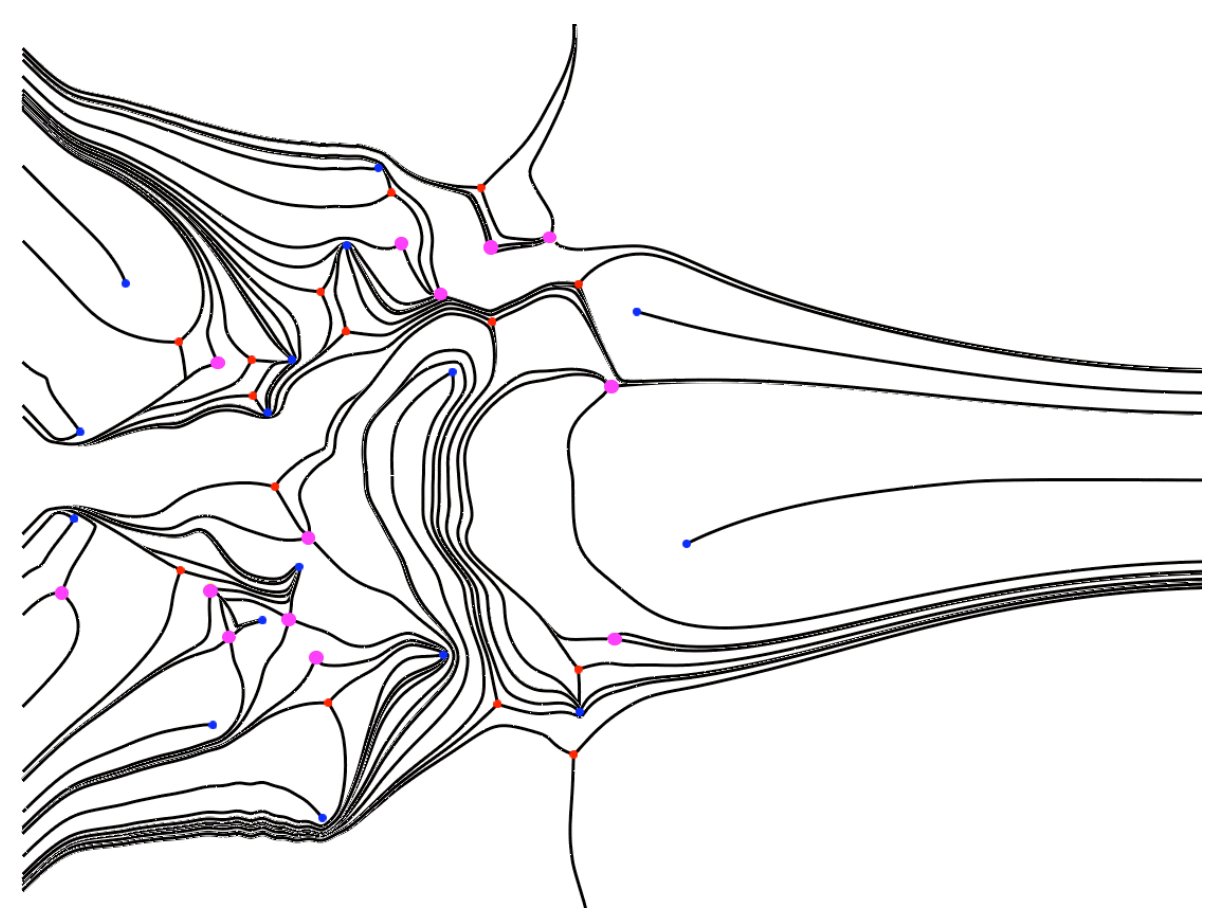

Fig. 8. Scaled topology (threshold $=0.2$ )

(Non-linear singularities are depicted by big dots.) Increasing the threshold (threshold $=0.4)$, we simplify the topology further. There are now 31 degenerate points (17 being non-linear) and 76 separatrices in the graph: See Fig. 9. The last stage of our scaling process is obtained with threshold $=0.8$. We stop here because the resulting graph already appears very coarse. This last topology has 15 degenerate points (only 2 of them are original ones!) and 42 separatrices. The result is shown in Fig. 10.

To focus on the local effect of the method on close singularities, we propose samples of a close-up corresponding to increasing thresholds in Fig. 11.

\section{Conclusion}

We have presented a method that scales the topology of symmetric, second-order planar tensor fields defined over curvilinear grids. Such a scaling is necessary when dealing with turbulent tensor fields because their structural complexity results in cluttered depictions with standard techniques which inconveniences interpretation. Our method basically replaces several close features of small scale by a single one, with non-linear structure, using their topological equivalence in the large. Close features are first supplied by a cell-based clustering strategy that is monitored by the proximity of the degenerate points contained in a same cluster. In a second step, the internal cell structure of each retained cluster is changed to produce the desired local 


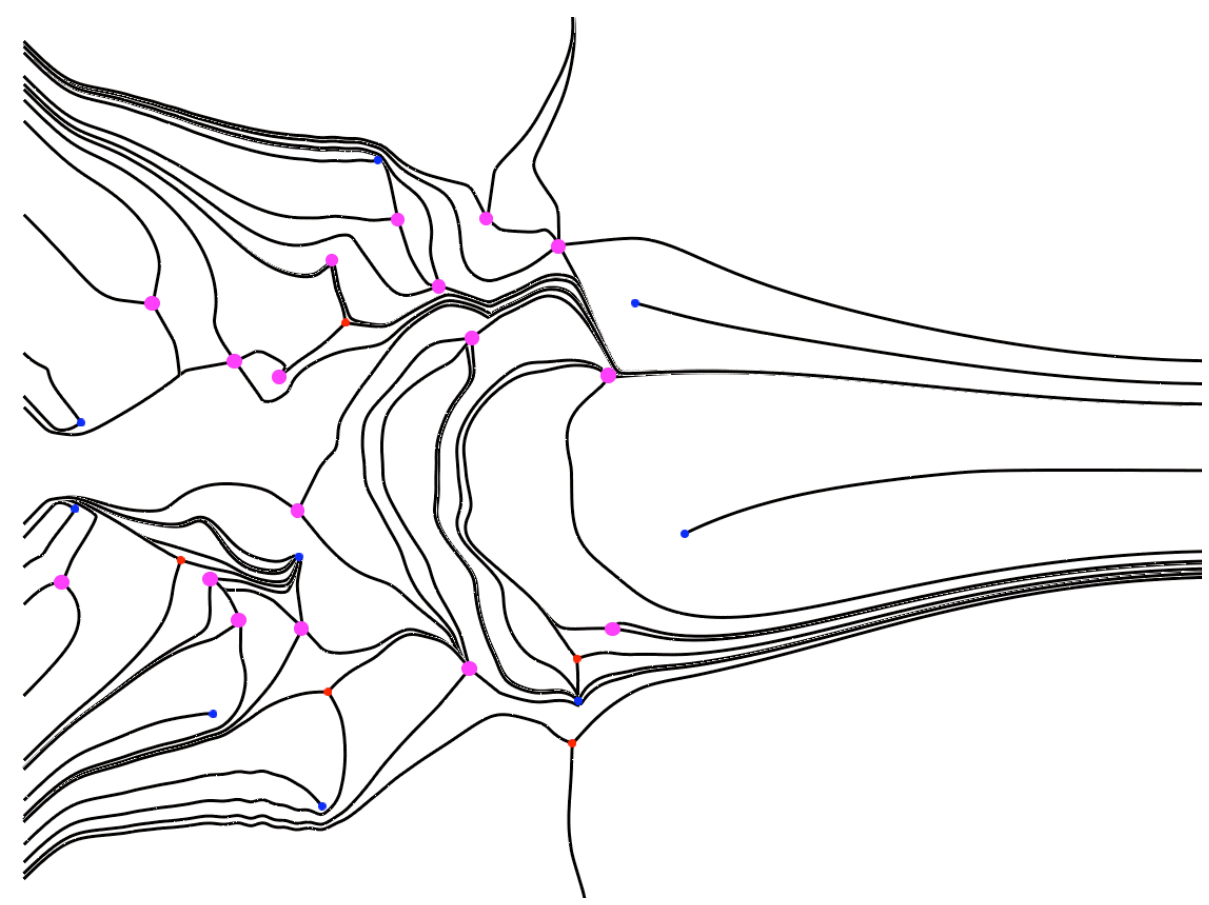

Fig. 9. Scaled topology (threshold $=0.4$ )

topological merging. At last, the resulting structure is identified mathematically. This eventually permits the clarification of the topological graph by the reduction of the number of singular points and associated separatrices. This was illustrated by our example, provided by a swirling jet simulation from the CFD: Global and local effects of the method were shown with increasing scaling factors.

\section{Acknowledgment}

The authors wish to thank Wolfgang Kollmann, MAE Department of the University of California at Davis, for providing the swirling jet dataset. Furthermore, we would like to thank Tom Bobach, Holger Burbach, Stefan Clauss, Jan Frey, Aragorn Rockstroh, René Schätzl and Thomas Wischgoll for their programming efforts.

\section{References}

[1] Delmarcelle T., Hesselink L., The Topology of Symmetric, Second-Order Tensor Fields. Proceedings IEEE Visualization'94, 1994.

[2] X. Tricoche, G. Scheuermann, H. Hagen, A Topology Simplification Method for $2 D$ Vector Fields. Proceedings IEEE Visualization'00, 2000. 


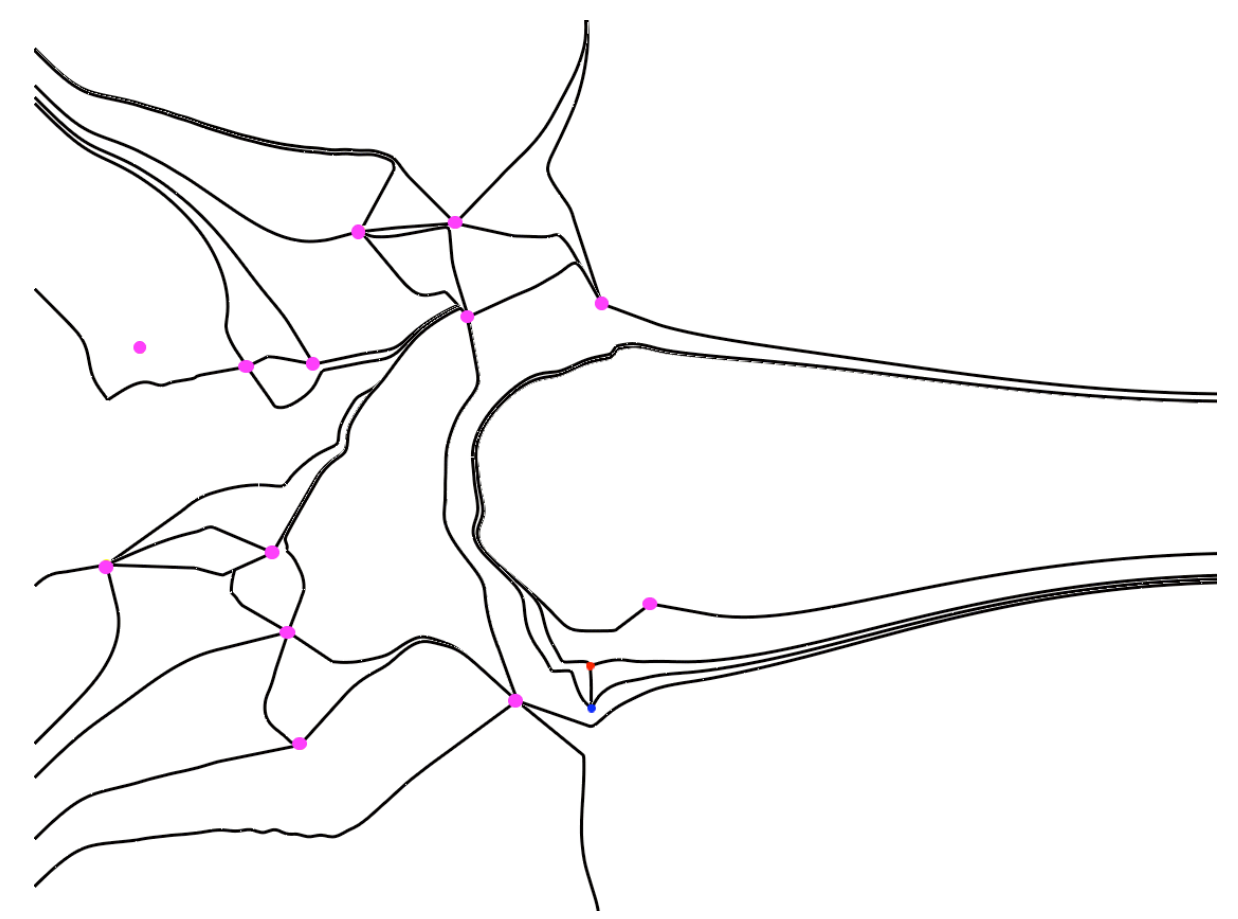

Fig. 10. Scaled topology (threshold $=0.8)$

[3] Lavin Y., Levy Y., Hesselink L., Singularities in Nonuniform Tensor Fields. Proceedings IEEE Visualization'97, 1997.

[4] Delmarcelle T., The Visualization of Second-Order Tensor Fields. PhD Thesis, Stanford University, 1994. 


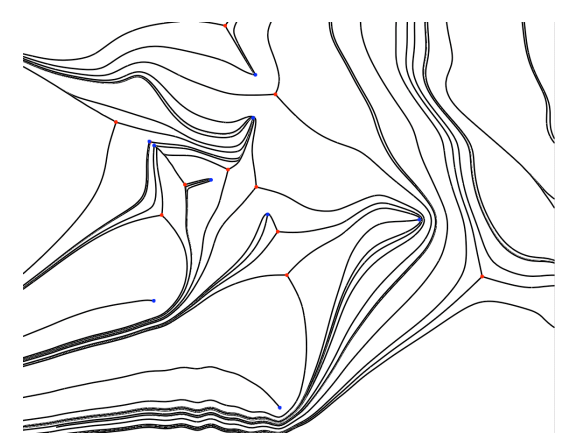

(a) Original topology

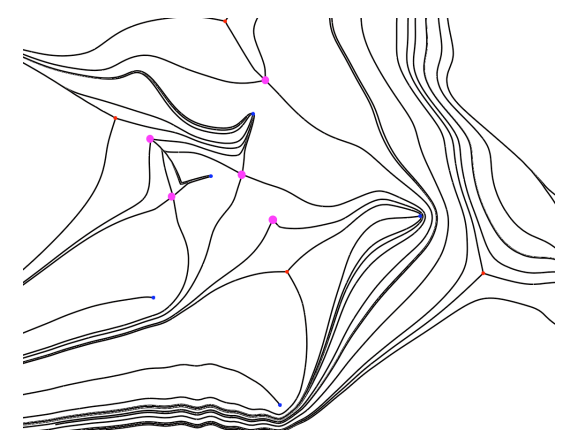

(c) Scaled topology (0.2)

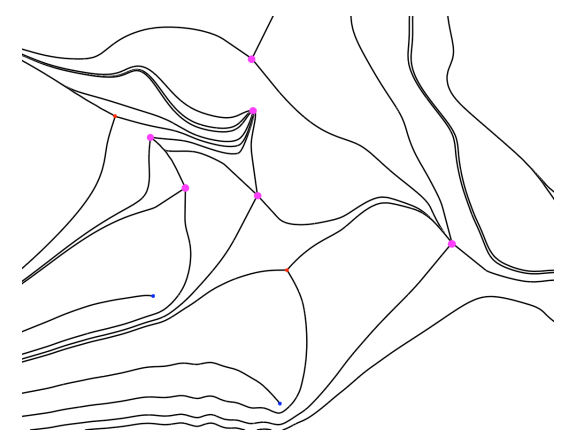

(e) Scaled topology $(0.45)$

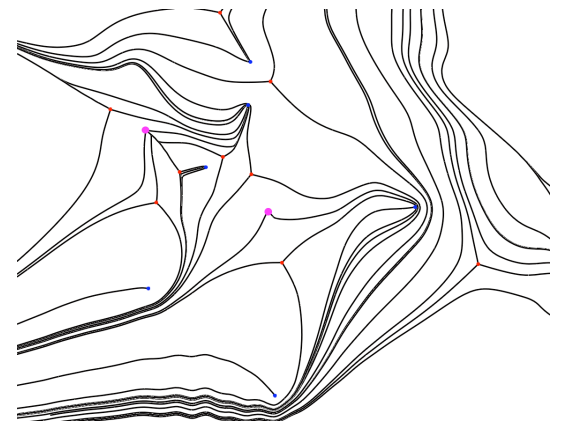

(b) Scaled topology (0.1)

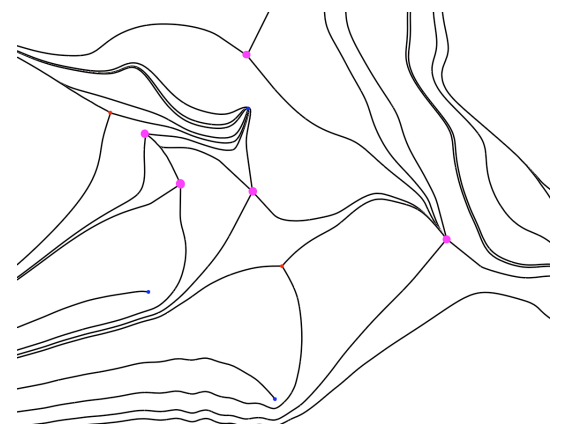

(d) Scaled topology (0.4)

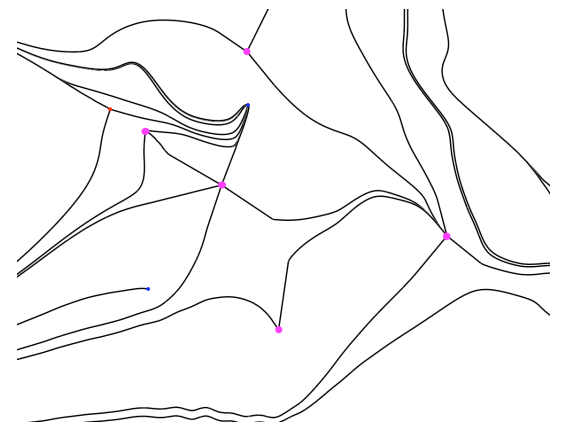

(f) Scaled topology (0.5)

Fig. 11. Samples of local topology scaling 